\title{
Scattered Point Cloud Data Reconstruction Algorithm Based on Local Convexity
}

\author{
Professor Dr. Sorush Niknamian \\ BSC in Applied Mathematics (IAUCTB), BSC in Mechatronics Engineering (SUT) \\ and $\mathrm{PhD}$ in Cell and Molecular Biology and Military Medicine, Liberty University \\ (LU), USA \\ Email: so.ninamian@gmail.com
}

\begin{abstract}
Point cloud data reconstruction is the basis of point cloud data processing. The reconstruction effect has a great impact on application. For the problems of low precision, large error, and high time consumption of the current scattered point cloud data reconstruction algorithm, a new algorithm of scattered point cloud data reconstruction based on local convexity is proposed in this paper. Firstly, according to surface variation based on local outlier factor (SVLOF), the noise points of point cloud data are divided into near outlier and far outlier, and filtered for point cloud data preprocessing. Based on this, the algorithm based on local convexity is improved. The method of constructing local connection point set is used to replace triangulation to analyze the relationship of neighbor points. The connection part identification method is used for data reconstruction. Experimental results show that, the proposed method can reconstruct the scattered point cloud data accurately, with high precision, small error and low time consumption.
\end{abstract}

Keywords: Local Convexity; Scattered Point Cloud; Data Reconstruction 


\section{INTRODUCTION}

Recently, the laser 3D imaging technology has developed rapidly, and the application scope of the 3D imaging system is also expanding ( $\mathrm{Yu}, 2017$; $\mathrm{Li}$ and $\mathrm{Kim}$, 2015). In order to meet the need of practical applications, various systems with different imaging methods emerge (Zhou, and Zhang, 2015). The demand for the precision of the point cloud data processing is getting higher and higher, and the point cloud data processing technology is developing continuously (Andrews et al., 2015). In the process of measurement, because there are no obvious geometric distribution in most cases and no obvious topological structure between data points with no organizational order, the whole point cloud presents a state of scattered and irregularities (Jia et al., 2016). The reconstruction of the scattered point cloud data has become the key subject for researchers (Zhang et al., 2016).

In the literature ( $\mathrm{Hu}$ et al., 2015), a scattered point cloud data reconstruction algorithm based on improved Ball Pivoting is proposed. For the existing problem of Ball Pivoting, the k-d tree is built for the initial point cloud data to improve the time efficiency of the searching area algorithm (Du and Hu, 2018; Lind et al, 2016; Sarikaya, 2018). By using the improved variable radius searching algorithm, the algorithm can process uneven point cloud data without generating hole (Bhavani and Jiji, 2018; Ezenwoke et al., 2018; Susanth and Kalayathankal, 2018). The grid topology is optimized. However, the time consumption of reconstruction with this method is high. In the literature (Tang et al., 2017), a tree reconstruction algorithm based on pint cloud data is proposed. Based on improved PC2Tree software, the 3D model of tree is reconstructed according to the topology of the skeleton point and the feature point. However, the reconstruction model obtained by this method is fuzzy and cannot be used for accurate identification. In the literature (Liu and 
Qin, 2017), a method of building facade reconstruction in data sparse region based on rule inference and point cloud data validation is proposed. Four neighborhood template matching method is used to reason the location and type of facade geometric structure of point cloud data sparse region. However, this method has not classified the point cloud data, which leads to the larger error of reconstruction (Lavanya, 2018).

To address the above problems, an algorithm of scattered point cloud data reconstruction based on local convexity in this paper. Experimental results show that, the proposed method can reconstruct the scattered point cloud data accurately, with high precision, small error and low time consumption. It can provide theoretical basis for the further research and development of the subject (Aragona et al, 2018; Chen et al, 2016; Ekman et al, 2017; Li and Wang, 2018; Lv, 2016; Mohammed and Sajjanhar, 2016; Yang et al, 2016).

\section{SCATTERED POINT CLOUD DATA RECONSTRUCTION ALGORITHM BASED} ON LOCAL CONVEXITY

\subsection{Scattered point cloud data preprocessing}

As the massive point cloud data is disorder and redundancy, the point cloud data is needed to be preprocessed before registration, including outlier identification and filtering. The current outlier identification methods include the density-based method, the distancebased method, the depth-based method, the distribution-based method, and the clusteringbased method (Li, Stoddart and Hitchens, 2017).

In this paper, a method of surface variation based local outlier factor (SVLOF) is proposed. The idea of the method is the outliers are to be found and divided into outliers far from the main entity and outliers near the main entity. The two kinds of outliers are 
classified and filtered. The 3D area growth method is used for far outlier identification and SVLOF is for analysis of near outlier.

A point $p$ and its point set $S$ are known. The under $k$ neighborhood $N b_{k}^{\alpha}$ of this point is the set of $k$ nearest points to the point $p$ in the point set $S$, which does not include the point $p$. The saturated $k$ neighborhood $N b_{k}^{\beta}$ is the set of $k+1$ nearest points to the point $p$ in the point set $S$, which includes the point $p$.

$$
N b_{k}^{\alpha}(p)=\left\{p_{i} \mid \sum_{i=1}^{k} p_{i}-p<\sum_{i=1}^{k} o_{i}-p, p_{i} \neq p, \forall o_{i} \in S, o_{i} \neq p\right\}
$$

$$
N b_{k}^{\beta}=\left\{N b_{k}^{\alpha}(p) \bigcup p\right\}
$$

where $q_{i}$ denotes the data point, $o_{i}$ denotes the center point of the microtangent plane fitted at the data point.

The eigenvalues of the covariance matrix of the point and its neighborhood can reflect the characteristics of local surface of the point. Based on this, the surface variation can be defined to identify the outlier (Nurunnabi, West and Belton, 2015).

The surface variation is defined by

$$
\sigma_{k}(p)=\frac{\lambda_{0}}{\lambda_{0}+\lambda_{1}+\lambda_{2}}
$$

where the eigenvalues of $\lambda_{0}, \lambda_{1}$, and $\lambda_{2}$ represent the variation of the data point on the axes of $\mathrm{x}, \mathrm{y}$, and $\mathrm{z}$. As the covariance matrix is a symmetric matrix, the eigenvalues are all 
greater than zero. $\sigma_{k} \in\left[0, \frac{1}{3}\right] . \sigma_{k}$ represents the deviation of the point from the tangent plane along the normal vector, also called as roughness. $\sigma_{k}=0$ represents the local point cloud data is on a plane. $\sigma_{k}=\frac{1}{3}$ represents point cloud data is with isotropic distribution.

If the point is an outlier, the surface variation of the point is large. However, the areas of corner and ridge also have larger surface variation, so the surface variation cannot be used as a unique identifier of outlier detection. Therefore, SVLOF is introduced to detect the outlier.

$$
\operatorname{SVLOF}_{k}(p)=\frac{\sigma_{k}^{\beta}(p)}{\sigma_{k}^{\alpha}(p)}
$$

where $\sigma_{k}^{\alpha}(p)$ is the surface variation of the under $k$ neighborhood of the point $p, \sigma_{k}^{\beta}(p)$ is the surface variation of the saturated $k$ neighborhood.

If the data point is a non-outlier point, the surface variation of the under $k$ neighborhood of the point is the same as the surface variation of the saturated $k$ neighborhood. If the difference of the surface variation is great, combining with the large SVLOF, the point is identified to be an outlier.

As the massive point cloud data is disorderly in this paper, the traditional method cannot be applied well. Therefore, a method of area growth based on distance query is proposed for the non-topological point cloud data. The steps of this method are as follows.

(1) Build an empty list $L$ and a new region point set $S_{i}$ ( $i$ is the number of points in the set). All points are labeled as the non-accessed state. The radius of the area growth is set to $r$. 
(2) Random select a point $p$ from the non-accessed point cloud. The point is pushed in the list $L$ and the set $S_{i}$, and then the point $p$ is labeled as a accessed point.

(3) The first element $p$ in the list $L$ is selected as growing candidate point to find the under $k$ neighborhood of the point $p$.

(4) The rest points unlabeled as accessed in the under $k$ neighborhood of $p$ are used in the list $L$ and the set $S_{i}$, and then labeled as accessed points.

(5) Delete the first element in the list $L$.

(6) When the list is empty and all points are labeled as accessed, the area growth is over, otherwise go on.

After filtering, the main entity of point cloud and near outlier is remained. The near outlier is needed to be identified.

Firstly, by using Eq. (1)-Eq. (3), the surface variation of each point in the point cloud data is calculated, and the points with larger surface variation are determined as the potential outlier.

Secondly, by using Eq. (4), SVLOF of all potential outliers are calculated. If the value of SVLOF is larger than the threshold, the point is considered as an outlier, and filtered. Outlier filtering is shown in Fig. 1.

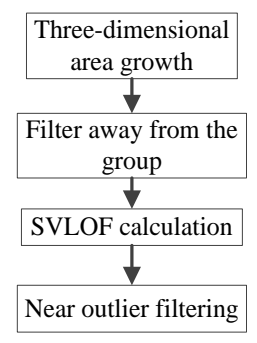




\section{Figure 1 The process of outlier filtering}

\subsection{Scattered point cloud data reconstruction algorithm based on local convexity}

The local convexity algorithm is mainly applied to urban environment data processing. The basic idea is to divide the data points by using the geometric characteristics between 3D data points. The points belonging to the same object is divided into the same area for reconstruction. The local convexity algorithm has the problems of poor detail processing and heavy computation. In this paper, the local convexity algorithm is improved. The method of constructing local connection point set is used to replace triangulation to analyze the relationship of neighbor points and the connection part identification method is used for data reconstruction. The complexity of the improved algorithm is reduced.

Local convexity is to measure the characteristics of the local surface around the two adjacent measured points, as shown in Fig. 2. Arbitrary two points $p_{i}$ and $p_{j}$, the displacement vector is $d_{i, j}=-d_{j, i}$. If the two normal vectors $n_{i}$ and $n_{j}$ are in the same direction or all points on any surface are under the other surface, the two points will satisfy the condition of local convexity, denoted as convexity value $c_{i, j}$. If the two point is to satisfy the local convexity, the value of $c_{i, j}$ is close to 1 , otherwise close to 0 .

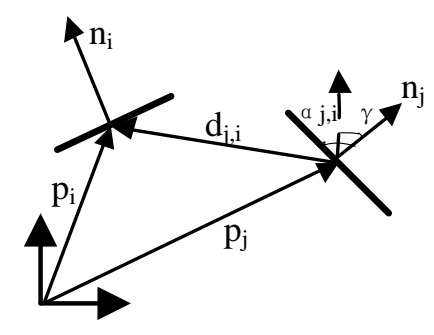




\section{Figure 2 Local convexity}

Convexity value $c_{i, j}$ is defined by

$$
c_{i, j}=\max \left\{\begin{array}{l}
\operatorname{sigm}\left(-n_{i}^{T} n_{j},-\cos \left(v_{n S \operatorname{sim}}\right), v_{n \operatorname{sinF} F}\right) \\
\operatorname{sigm}\left(\max \left\{\frac{n_{i}^{T} d_{i, j}}{\left\|d_{i, j}\right\|}, \frac{n_{j}^{T} d_{j, i}}{\left\|d_{j, i}\right\|}\right\}, \cos \left(90^{\circ}-v_{\text {conv }}\right), v_{\text {conv }}\right)
\end{array}\right\}
$$

The dot product of the surface normal vector $n_{i}$ and $n_{j}$ is to measure whether the direction is the same. If the result is equal to 1 , it represents the direction is the same. If the result is equal to 0 , it represents the direction is opposite. $v_{n S \operatorname{sim}}$ and $v_{n \operatorname{sinF}}$ is a constant, respectively. $v_{n S \text { Sim }}$ is normal similarity soft threshold, which is used to compare the angle between noise and normal vector. $v_{n S \sin F}$ is the tangent slope at $v_{n S \operatorname{sim}}, v_{\text {conv }}$ and $v_{\text {conv }}$ are constants, SumLI is convexity soft threshold, which is the allowed maximum angle of the other points above the surface. $v_{\text {conv }}$ is the tangent slope at $v_{\text {conv }}$. As the function sigm is monotone decreasing, in order to ensure the maximum value when the normal directions is consistent, minus is added in the function sigm.

$\operatorname{sigm}(x, \theta, m)$ is the soft threshold of S-type function (Klyuzhin and Sossi, 2017), which is given by

$$
\operatorname{sigm}(x, \theta, m)=0.5-\frac{0.5(x-\theta) m}{\sqrt{1+(x-\theta)^{2} m^{2}}}
$$

where $\theta$ is the effective threshold, $m$ is range parameter affecting the tangent slope at the threshold. 
The process of depth image processing is as follows. Firstly, the point cloud data detected by $3 \mathrm{D}$ imaging system is projected as the pixel of depth image by using projection raster method (Dougan, 2015). When the 3D point cloud data is converted to the depth image, any 3D point is required to correspond to the pixel $(u, v)$ of the depth image with the depth $r_{(u, v)}$. It should be noted that 3D imaging system may have insufficient resolution in data collection. When the measured point is projected to the pixel, there will be fuzzy. It can be solved by discarding all the measured points except the points nearest the pixel. Secondly, each pixel in the obtained depth image is backprojected to the 3D point cloud data. So the adjusted point cloud data can be filtered out of some redundant points compared with the previous scattered point cloud and the pixel $(u, v)$ has the information of distance $r$.

Because the neighbor points in the $3 \mathrm{D}$ point cloud do not necessarily belong to the same object, the boundary is needed to be decided to determine whether the local connection points belong to the same object, which is obtained with discontinuities of depth values. For any two neighbor pixels $i$ and $j$, the connection value is used to determine the boundary. If $l_{i, j}$ is close to 1, it represents the two neighbor pixels probably belong to the same part, otherwise, if it is close to 0 , it is more impossible to belong to the same part.

If the absolute variation of depth values of two neighbor pixels and the relative variation of depth values of left neighbor pixel and right neighbor pixel are limited, the two pixels belonging to the same part. Therefore, In the four ordered neighbor pixels, the connection value $l_{i, j}$ is influenced by three variables of $\left(r_{i}-r_{j}\right),\left(r_{i}-r_{j}\right)-\left(r_{h}-r_{i}\right)$, and $\left(r_{i}-r_{j}\right)-\left(r_{j}-r_{k}\right)$. Only when three variables are relatively small, there is no boundary in $i$ and $j$. 
By using the Fuzzy logic method, $l_{i, j}$ is defined by

$$
l_{i, j}=\min \left\{\operatorname{sigm}\left(\left|\frac{\left(r_{i}-r_{j}\right)}{\min \left\{r_{i}, r_{j}\right\}}\right|, v_{r D i f f}, \frac{2}{v_{r D i f f}}\right), \operatorname{sigm}\left(\left|\frac{\left(r_{i}-r_{j}\right)-\left(r_{h}-r_{j}\right)}{\left(r_{h}-r_{j}\right)}\right|, v_{r N D i f}, v_{r N F}(r)\right), \operatorname{sigm}\left(\left|\frac{\left(r_{i}-r_{j}\right)-\left(r_{j}-r_{k}\right)}{\left(r_{j}-r_{k}\right)}\right|, v_{r N D i f f}, v_{r N F}(r)\right)\right\}
$$

where, $v_{r D i f f}$ and $v_{r N D i f f}$ are constants, $v_{r D i f f}$ is the soft threshold of depth variation, $v_{r N D i f f}$ is the soft threshold of relative variation of depth value of left neighbor pixel, $v_{r N F}(r)$ is the tangent slope at $v_{r N D i f f}$.

After obtaining the local connection point set by analyzing the relationship of neighbor points, so it is necessary to judge reconstruction standard for the point in local connection point set. The reconstruction standard is given by

$$
s_{i, j}=\left\{\begin{array}{cc}
\text { ture } & \text { if } c_{i, j} \cdot l_{i, j} \geq v_{s t} \\
\text { false } & \text { else }
\end{array}\right.
$$

where $v_{s t}$ is the threshold of reconstruction standard. $s_{i, j}$ is symmetric and $s_{i, j}=s_{j, i}$. If the value of $s_{i, j}$ is ture, it represents the points $p_{i}$ and $p_{j}$ belong to the same part.

In the original algorithm, the computation of pred is also used to reconstruct the data points. The computation of pred is given by

$$
\operatorname{pred}\left(\delta_{g}\right)=\text { ture } \quad \text { iff } \forall i, m \in \delta_{g} \exists j, \cdots, l \in \delta_{g}: s_{i, j} \wedge \cdots \wedge s_{l, m}=\text { ture }
$$

where $\delta_{g}$ is the set of point cloud data to be reconstruction, $i, j, m, \cdots, l$ is the data point in the set. 


\section{EXPERIMENTAL RESULTS AND ANALYSIS}

Several point cloud data model is selected for experiments. The experiments environment is with Intel I5 (3210 M, 2.50 GHz) CPU, 4 GB memory, and Windows 7 (64 bit). Through multiple data testing and reconstruction effect analysis, the experimental parameters are optimized. The parameters are set as follows. $v_{n s i m}=14.8062^{\circ}, v_{\text {conv }}=-7.67^{\circ}$, $v_{\text {nSimF }}=10, v_{\text {convF }}=1.154, v_{r D i f f}=0.136, v_{r N D i f f}=1.90154$, and $v_{r S B}=10.03 m$. The value of $v_{s t}$ ranges from 0.25 to 0.55 , which is adjusted to achieve the best effect of reconstruction.

The reconstructed mesh density is obtained in the cases of different thresholds of reconstruction standard, as shown in Fig. 3.

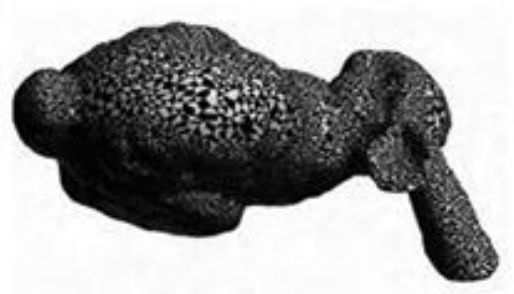

(a) Reconstruction standard threshold is 0.3

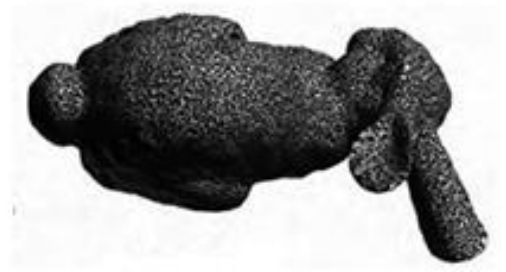

(b) Reconstruction standard threshold is $\mathbf{0 . 3 5}$ 


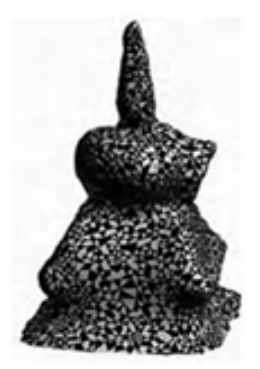

(c) Reconstruction standard threshold is 0.4

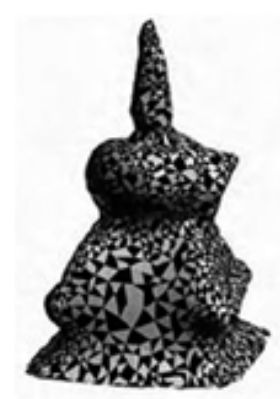

(d) Reconstruction standard threshold is $\mathbf{0 . 5}$

Figure 3 Reconstruction results obtained with different reconstruction standard thresholds

From Fig. 3, when the reconstruction threshold standard is different, the reconstructed mesh density is different. When the reconstruction standard threshold is 0.35 , the mesh density is the largest, which ensures the accuracy of reconstruction. Therefore, the reconstruction effect of scattered point cloud data is the best.

Fig. 4 shows the scattered point cloud data model with preprocessing and without preprocessing. Fig. 4(a) shows the reconstruction of sculpture model without preprocessing while Fig. 4(b) with preprocessing. Fig. 4(c) shows the reconstruction of squirrel model without preprocessing while Fig. 4(d) with preprocessing. 


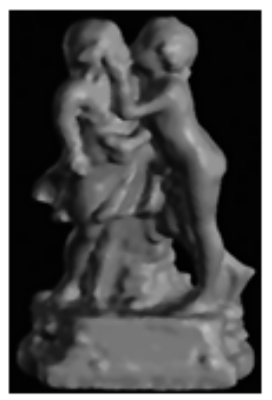

(a) Reconstruction of sculpture model without preprocessing

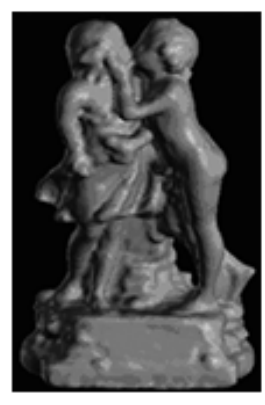

(b) Reconstruction of sculpture model with preprocessing

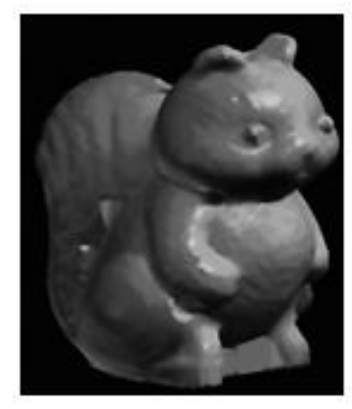

(c) Reconstruction of squirrel model without preprocessing 


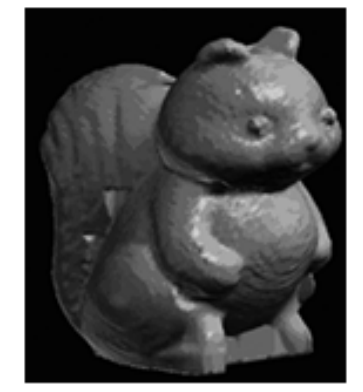

(d) Reconstruction of squirrel model with preprocessing

Figure 4 Reconstruction results of the scattered point cloud data model before and after preprocessing

From Fig. 4, after preprocessing the scattered point cloud data, the reconstructed image has a high resolution and high accuracy. As the proposed method uses SVLOF to filter and smooth the scattered point cloud data, the noise in scattered point cloud data is reduced to ensure the accuracy of reconstruction.

To verify the precision of the proposed method, the experiment of the parameters of time of modeling and extraction are carried out. The experimental results are shown in Table 1.

Table 1 Comparison of reconstruction parameters before and after preprocessing of the scattered point cloud data

\begin{tabular}{lllllll}
\hline Model & $\begin{array}{l}\text { Data before } \\
\text { preprocessi } \\
\text { ng/MB }\end{array}$ & $\begin{array}{l}\text { Data after } \\
\text { preprocessi } \\
\text { ng/MB }\end{array}$ & $\begin{array}{l}\text { Model } \\
\text { time/s } \\
\text { Modeling }\end{array}$ & reconstruction & $\begin{array}{l}\text { Extraction } \\
\text { error/mm }\end{array}$ & $\begin{array}{l}\text { Average } \\
\text { error/mm }\end{array}$ \\
\hline Sculpture & 712 & 712 & 0.157 & 3.869 & 0.157 & 0.203 \\
Squirrel & 2078 & 2078 & 0.643 & 7.845 & 1.021 & 0.937 \\
\hline
\end{tabular}

From Table 1, the proposed method can quickly reconstruct the scattered point cloud data, and the reconstruction error is small. 


\section{CONCLUSIONS}

In recent years, laser 3D imaging technology has been developing rapidly, becoming more mature, and expanding its application scope. It has been applied to many fields, such as digital city, reverse engineering and so on. The data collected by the laser 3D imaging system is a large number of scattered point cloud data. The separation of the objects with different attributes needs the point cloud data reconstruction. Therefore, the point cloud data reconstruction is the key part of the point cloud data processing, and provides the basic information for the classification and recognition. A scattered point cloud data reconstruction algorithm based on local convexity is proposed in this paper. Experimental results show that, the proposed method can reconstruct the scattered point cloud data accurately, with high precision, small error, and low time consumption.

\section{ACKNOWLEDGEMENTS}

Project supported by special funds of Guangdong province science and technology development project "the Intelligent Vocational Education Great data Engineering Technology Research Center of Guangdong Province (No. 706049150203)", and the third batch Guangzhou education bureau academic team innovation project "complex visual scene of pedestrian detection and tracking of academic innovation team (No. 1201610034)”.

\section{REFERENCES}

Andrews, B., Chen, X., Fang, H., et al. (2015). Expansion of co-compact convex spacelike hypersurfaces in minkowski space by their curvature. Indiana University Mathematics Journal, 64(2), 635-662. DOI: 10.1512/iumj.2015.64.5485. 
Aragona, R, Rimoldi, ASala, M. (2018). A note on an infeasible linearization of some block ciphers. Journal of Discrete Mathematical Sciences and Cryptography, 21(1): 209-218.

Bhavani, R.R. and Jiji, G.W. (2018). Image registration for varicose ulcer classification using knn classifier. International Journal of Computers and Applications, 40(2), 88-97.

Chen, L, Xia, X, Zheng, H, Qiu, M. (2016). Friction torque behavior as a function of actual contact angle in four-point-contact ball bearing. Applied Mathematics and Nonlinear Sciences, 1(1): 53-64.

Dougan, J.N. (2015). Spatial data acquisition with unmanned aerial system and evaluation of photogrammetric point cloud classification quality. Nutrition, 31(1), 64-71.

Du, P, Hu, H. (2018). Intelligent detection algorithm of dissolved organic nitrogen in water environment protection of forest park. Journal of Discrete Mathematical Sciences and Cryptography, 21(2): 239-244.

Ekman, J, Hietaniemi, M, Kolari, M, Nelson, MKeegan, K. (2017). Novel starch saving programme improves sustainability in board manufacturing through the reuse of recycled fibre starch. Paper Asia, 33(6): 18-22.

Ezenwoke, A.; Daramola, O., and Adigun, M. (2018). Qos-based ranking and selection of saas applications using heterogeneous similarity metrics. Journal of Cloud Computing, 7(1)

Hu, S.L., Zhou, M.Q., Shui W.Y., et al. (2015). Improved Pivoting Ball Algorithm for Nonuniform Point Cloud Data. Journal of System Simulation, 27(10), 2446-2452. DOI: 10.16182/j.cnki.joss.2015.10.033.

Jia, B., Wee, T.L., Boudreau, C.G., et al. (2016). Parallelized cytoindentation using convex micropatterned surfaces. Biotechniques, 61(2), 73. DOI: 10.2144/000114436. 
Klyuzhin, I., and Sossi, V. (2017). Pet image reconstruction and deformable motion correction using unorganized point clouds. IEEE Transactions on Medical Imaging, PP. (99), 1-1. DOI: 10.1109/TMI.2017.2675989.

Lavanya, B. (2018). Unsteady mhd convective laminar flow between two vertical porous plates with mass transfer. Journal of Mechanical Engineering Research and Developments, 41(1): 97-109.

Li, F., Stoddart, D., and Hitchens, C. (2017). Method to automatically register scattered point clouds based on principal pose estimation. Optical Engineering, 56(4), 044107. DOI: 10.1117/1.OE.56.4.044107.

Li, X, Wang, J. (2018). Parallel machines scheduling based on the impact of deteriorating maintenance. Journal of Interdisciplinary Mathematics, 21(3): 729-741.

Li, Y., and Kim, J. (2015). Fast and efficient narrow volume reconstruction from scattered data. Pattern Recognition, 48(12), 4057-4069. DOI: 10.1016/j.patcog.2015.06.014.

Lind, T, Yan, H, Jappinen, H. (2016). Sustainability and energy efficiency in the pulp and paper industry. Paper Asia, 32(5): 15-21.

Liu, Y.W., and Qin, S.S. (2017). Building Facade Reconstruction on Sparse LiDAR Data Region. Journal of Applied Sciences, 35(2), 217-225. DOI: 10.3969/j.issn.02558297.2017.02.008.

Lv, H. (2016). Characteristics and mechanical properties of rolling-element bearings. International Journal of Vehicle Structures and Systems, 8(3): 161-166. 
Mohammed, AA, Sajjanhar, A. (2016). Experimental comparison of approaches for feature extraction of facial attributes. International Journal of Computers and Applications, 38(4): 187-198.

Nurunnabi, A., West, G., and Belton, D. (2015). Outlier detection and robust normal-curvature estimation in mobile laser scanning 3d point cloud data. Pattern Recognition, 48(4), 1404-1419. DOI: 10.1016/j.patcog.2014.10.014.

Sarikaya, MZ. (2018). On fejer type inequalities via fractional integrals. Journal of Interdisciplinary Mathematics, 21(1): 143-155.

Susanth, C. and Kalayathankal, S.J. (2018). Operations on independence numbers of certain graph classes. Journal of Discrete Mathematical Sciences and Cryptography, 21(1), 7582.

Tang, L.Y., Zhang, H., Huang, H.Y., et al. (2017). Improved Method for 3D Reconstruction of Tree Model Based on Point Cloud Data. Transactions of the Chinese Society for Agricultural Machinery, 48(2), 186-194. DOI: 10.6041/j.issn.1000-1298.2017.02.025.

Yang, Z, Xie, J, Zhang, L, Guo, M, Ran, J. (2016) Experimental study and kinetic analysis of cuo/-al2o3absorbed so2. International Energy Journal, 16(4): 173-182.

Yu, G.Z. (2017). Simulation of 3D Point Cloud Data Reconstruction Based on White Noise. Computer Simulation, 34(5), 444-447. DOI: 10.3969/j.issn.1006-9348.2017.05.097.

Zhang, M., Wu, T., Beeman, S.C., et al. (2016). Efficient small blob detection based on local convexity, intensity and shape information. IEEE Transactions on Medical Imaging, 35(4), 1127-1137. DOI: 10.1109/TMI.2015.2509463. 
Zhou, A., and Zhang, Y. (2015). Explicit integration scheme for a non-isothermal elastoplastic model with convex and nonconvex subloading surfaces. Computational Mechanics, 55(5), 943-961. DOI: 10.1007/s00466-015-1144-3. 